\title{
Characterization of Impacts of Elastic-plastic Spheres
}

\author{
Bence Szabó1, Attila Kossa ${ }^{1 *}$ \\ ${ }^{1}$ Department of Applied Mechanics, Faculty of Mechanical Engineering, Budapest University of Technology and Economics, \\ 1111 Budapest, Múegyetem rkp. 5., Hungary \\ *Corresponding author, e-mail: kossa@mm.bme.hu
}

Received: 10 January 2020, Accepted: 25 March 2020, Published online: 08 April 2020

\begin{abstract}
This work presents explicit dynamic finite element simulations of various impacts of elastic-plastic solid spheres with flat walls. Different analytical models describing the mechanics of the impact phenomenon are also presented. Elastic and elastic-plastic material models for the sphere and the wall are considered during the analyses. The applicability of these different models is demonstrated and their accuracies are investigated. Closed-form analytical functions are proposed to describe the relationship between the initial velocity of the sphere and the investigated contact characteristics for the given material models. Analysis is carried out to study the effect of the friction coefficient as well as the angle of impact for various cases.
\end{abstract}

\section{Keywords}

impact, finite element analysis, contact force

\section{Introduction}

There exist several simple models to characterize the mechanics of impact of elastic bodies. The elementary models usually employ purely elastic material behavior.

The simplest model describes the maximal contact force with the help of a linear spring, where the spring represents the elasticity of the bodies in contact [1]. A widely used model is the nonlinear Hertz model [2, 3], which utilizes the maximal indentation and a parameter that depends on the shapes and material properties of the contacting bodies to describe the maximal contact force. It should be emphasized, that this model also provides a closed-form formula for the calculation of the contact time.

Hunter [4] has shown that during elastic impact of a sphere and a wall, a small portion of the kinetic energy is dissipated. He proposed a model that incorporates this effect into the Hertz model, in which the indentation is approximated as a half sine wave and the contact force is described with the help of the Newton's second law of motion.

Based on the model suggested by Hunter [4], Reed [5] introduced an enhanced model, in which the indentation - time function of Hunter was directly substituted into the normal force - indentation function described by Hertz [3]. The energy dissipation given by this model is significantly larger than in case of the Hunter model.
Several other models were also proposed, that incorporate energy dissipations. These models in most cases describe the dissipation with the help of an inner damping model.

One of the first models belonging to this class was introduced by Kelvin and Voigt (cited in Goldsmith's work [6]). They modeled the total force with a linear spring and a linear dashpot connected in parallel networks. The part of the expression related to the spring is the elastic force component and the part corresponding to the dashpot takes the energy dissipation into account. This model has some drawbacks, namely that at the first moment of the contact, the contact force is not zero as a result of the part related to the dashpot. Furthermore, at the end of the restitution phase, the relative velocity is negative and the indentation is zero leading to negative force.

Hunt and Crossley [7] introduced a new improved model and they clearly explained the limitations and physically unrealistic behavior of the elementary Kelvin and Voigt model. Their model describes the contact force using the Hertz model in combination with a nonlinear dashpot. Hunt and Crossley reported the value of the damping coefficient that satisfies the conditions at the initial moment of contact and at the time of the separation of the contacting bodies. The model is mostly applicable in case of impacts, where the coefficient of restitution is close to unity. 
The previous dissipative contact models can be used in cases, where the area of contact is relatively small compared to the size of the bodies in contact. To overcome this limitation, Gonthier et al. [8] proposed a model, which is similar to the model of Hunt and Crossley with a different damping coefficient.

One of the most recent dissipative models was introduced by Flores et al. [9], who proposed another nonlinear damping characterization. The last two models represent a larger damping behavior, which provide more accurate results at moderate velocity impacts, which can be described by a lower coefficient of restitution.

Besides the elastic material models, several other models were introduced, where the elastic-plastic behavior of the materials was also taken into account, as in most cases there are permanent deformations during impacts. In order to simplify the problem and the characterization, only one of the contacting bodies was modelled using elastic-plastic material model. Based on this, the contact models can be divided into two main groups. One of them is the flattening models, where the sphere has been considered to be elastic-plastic material, whereas the wall is modeled as rigid body. The other group is the collection of indentation models, where the sphere has been considered rigid and the wall to be deforming material.

The topic of this article is closer to the first group, namely the flattening models. In this case, the research studies demonstrated that after a certain value of the contact force, the pressure distribution is almost constant throughout the contact area. This was later verified by finite element calculations $[10,11]$.

Thornton [12] proposed a model in which the entire impact was divided into three parts; an elastic indentation, a plastic indentation and an elastic restitution. According to this assumption the contact force during the plastic phase after the Hertzian elastic phase is tangent to the curve described by Hertz, as Thornton and Ning [13] presented.

There can be found a few models describing the case of the impact of an elastic plastic sphere and an elastic plastic wall for instance [14]. However, these models are more complex and require numerous parameters. In addition, most of them are based on finite element calculations. Therefore, the finite element analysis has an important role in the investigation of these cases.

The advanced models and the comparison of different material models could help to develop various applications of impacts in other fields, such as impulse excitation as it is demonstrated in [15].
The impact characteristics of a solid sphere and a flat wall are investigated in this paper in case of different material model pairs. The applicability of the material models as a function of the initial velocity magnitude of the sphere is examined. Moreover, the effect of friction coefficient and angle of impact are also considered.

The investigated material models and the results obtained by them are given in Section 2. Then the comparison of the results for the different material models is presented in Section 3. In Section 4, the effect of the friction coefficient is investigated. In Section 5, the effect of the angle of impact is studied.

\section{Finite element models}

The impact of a solid sphere with diameter $d=6 \mathrm{~mm}$ and a flat wall is considered. The following material parameters were used in the simulations: Young's modulus $E=208 \mathrm{GPa}$, Poisson's ratio $v=0.3$, density $\rho=7830 \mathrm{~kg} / \mathrm{m}^{3}$, yield stress $\sigma_{y}=882 \mathrm{MPa}$. The material properties were chosen based on [16]. Please note that the investigation of the material properties was not in the scope of this study. Three different material model pairs were investigated in the calculations:

1. Elastic sphere and rigid wall (E-R),

2. Elastic sphere and elastic wall (E-E),

3. Elastic-plastic sphere and elastic-plastic wall (EP-EP).

In case of the EP-EP model, linear elastic perfectly plastic material model was applied.

The investigated contact characteristics were the maximal contact force and the contact time, and their dependence on the initial velocity of the sphere before the impact as the main parameter. Furthermore, the effect of the friction coefficient and the angle of impact were also analysed. Please note that the spinning of the sphere was not considered as the kinetic energy from the rotation is negligible compared to the kinetic energy related to the velocity.

The finite element simulations were performed in the commercial FE software ABAQUS [17]. The investigated parameters used in the analyses are listed in Table 1. $v$ represents the impact velocity, $\theta$ denotes the angle of impact, whereas $\mu$ is the friction coefficient between the ball and the wall.

The nature of the problem provides the opportunity to simplify the model and use axisymmetric model. The full 3D model and the axisymmetric model were compared in case of the EP-EP material model in order to confirm the validity of the axisymmetric approach. The obtained result can be seen in Fig. 1. The graphs clearly demonstrate 
Table 1 The investigated parameters

\begin{tabular}{lcc}
\hline$v[\mathrm{~m} / \mathrm{s}]$ & $\theta\left[^{\circ}\right]$ & $\mu[1]$ \\
\hline 1 & 0 & 0 \\
2 & 10 & 0.1 \\
5 & 30 & 0.4 \\
10 & 45 & 0.8 \\
20 & 60 & \\
30 & & \\
40 & & \\
50 & & \\
60 & & \\
80 & & \\
\hline
\end{tabular}
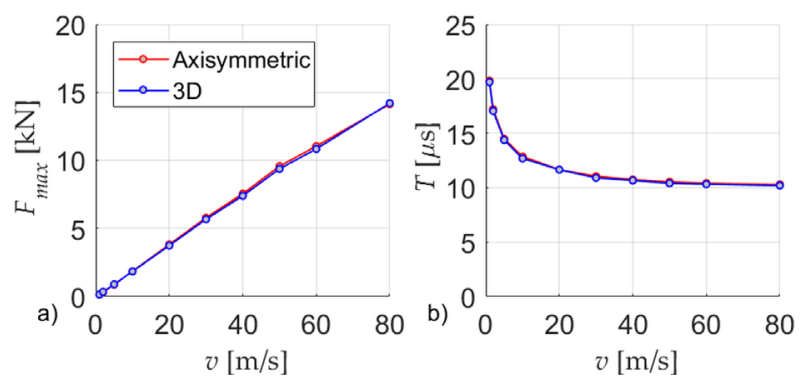

Fig. 1 The a) maximal contact force and b) contact time as the function of the velocity of the sphere before impact in case of the three dimensional and axisymmetric models

that the differences in the results are negligible, therefore the axisymmetric model can be used to obtain adequate results with significantly less computational time.

Moreover, a mesh independency analysis was also carried out. It was performed by creating 7 different mesh densities, each with a different number of elements, however the structure of the mesh was kept self-similar. The effect of the mesh size was investigated in case of the elastic sphere rigid wall model and when both parts were elastic plastic, respectively. The contact characteristics were monitored. The results showed that the contact characteristics do not change significantly above a certain number of elements in the mesh. Based on these results, the meshes were created in such a way that the number of elements does not affect the results and the computational time is kept minimal. It should be noted that in case of the elastic sphere elastic wall impact simulations the same mesh strategy and density were used as in case of the elastic-plastic model. This approach is based on the assumption that if the mesh is applicable in a more complex case then it will also be appropriate to use in a simpler scenario.

Furthermore, in order to validate the FE model and the simulation results, the obtained results in case of the E-E material model were compared with the Hertz contact theory [2] that describes the contact force during a quasi-static elastic contact of a sphere and an elastic half-plane. The numerical Hertzian dimensionless forcetime function has an analytical approximation given by the work of Reed [5].

The compared results can be seen in Fig. 2, where $F_{\text {max }}$ is the maximal contact force and $T$ denotes the contact time in case of the corresponding model. The results obtained from the finite element simulations show excellent agreement with the theoretical approximation, therefore the FE model can be considered validated.

\subsection{Elastic-rigid (E-R) model}

In the case of the first investigated material model pair an elastic sphere and a rigid wall was considered with frictionless normal impact. The applied finite element model is illustrated in Fig 3.

The investigated characteristics obtained from the simulations are presented in Figs. 4 and 5. The numerical results are approximated with the following closed-form analytical functions based on a standard curve-fitting procedure:

$$
\begin{aligned}
& F_{\text {max }}=0.1919 v^{1.24}, \\
& T=15.6324 v^{-0.2115} .
\end{aligned}
$$

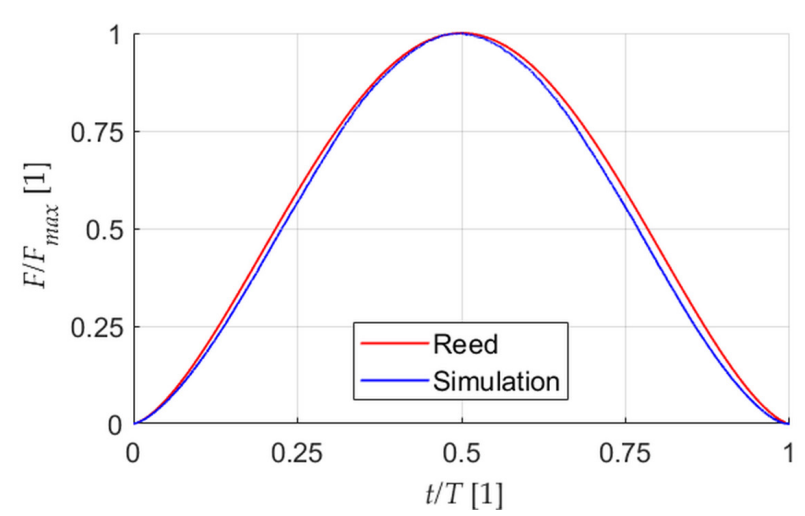

Fig. 2 The dimensionless force-time function obtained by FE simulation compared with the Hertzian function provided by Reed

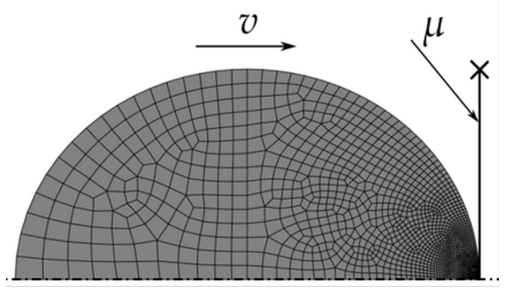

Fig. 3 The applied FE model in case of the E-R material model 


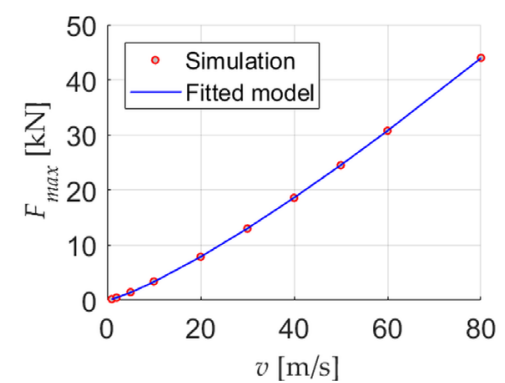

Fig. 4 The maximal contact force as the function of the velocity of the sphere before the impact

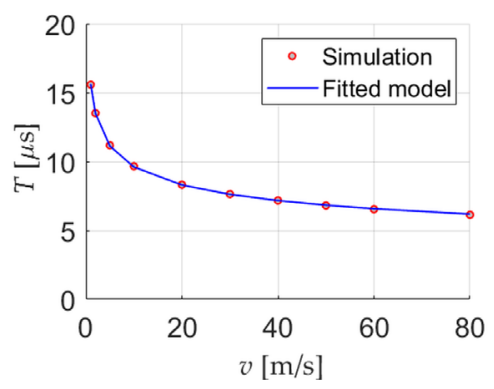

Fig. 5 The contact time as the function of the velocity of the sphere before the impact

The comparisons of the simulations and the fitted functions can also be seen in the figures. The fitted functions describe accurately the maximal contact force and the contact time along the entire investigated domain.

\subsection{Elastic-elastic (E-E) model}

In the second case, the frictionless normal impact of an elastic sphere and an elastic wall was investigated. The FE model applied in this case is presented in Fig. 6.

The obtained contact characteristics for this case can be seen in Figs. 7 and 8. With the help of the same curve fitting method as in the previous sub-section, the relation between the contact characteristics and the velocity before the impact was obtained as:

$$
F_{\max }=0.1683 v^{1.1962} \text {, }
$$

$$
T=20.6017 v^{-0.2083} \text {. }
$$

Several models are presented in the literature for the case of the impact of elastic bodies, from which the model of Hertz is the most widely used. Therefore, the contact characteristics described by the Hertz model is also plotted in the figures. The FE results show great similarity with the ones obtained by the Hertz model.

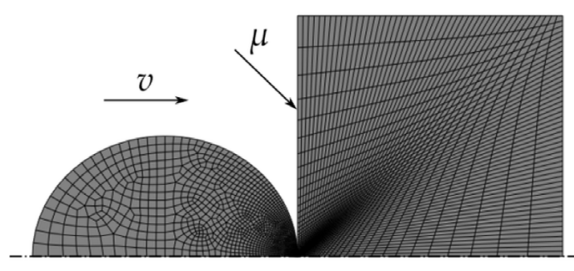

Fig. 6 The applied FE model in case of the E-E and EP-EP material models

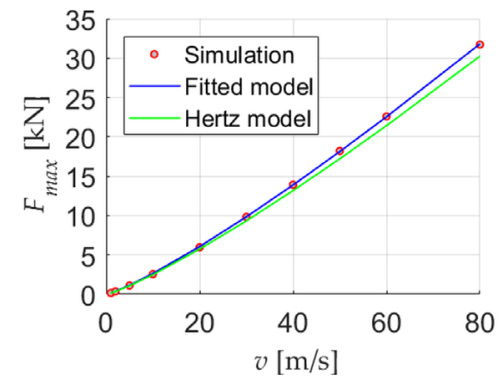

Fig. 7 The maximal contact force as the function of the velocity of the sphere before the impact obtained by the FE simulation and the results given by the fitted model and the Hertz model

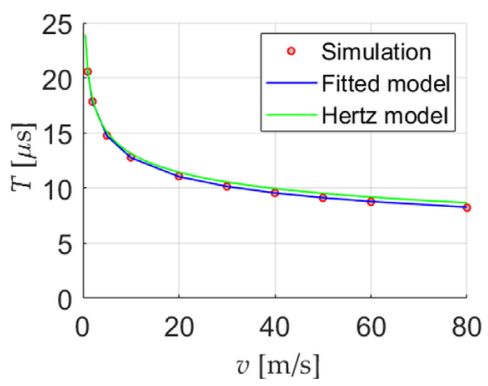

Fig. 8 The contact time as the function of the velocity of the sphere before the impact obtained by the FE simulation and the results given by the fitted model and the Hertz model

\subsection{Elastic-plastic - Elastic-plastic (EP-EP) model}

In this analysis, both the sphere and the wall were modeled with elastic-plastic material model with no hardening. The applied FE model is the same as in the previous sub-section, except the material model.

In this case, the function fitted to the results of the contact time was sought in a slightly more complex form. As a result, the relations can be given as

$$
\begin{aligned}
& F_{\text {max }}=0.236 v^{0.938}, \\
& T=7.7479+12.1336 v^{-0.3736} .
\end{aligned}
$$

The finite element results and the fitted curves can be seen in Figs. 9 and 10. 


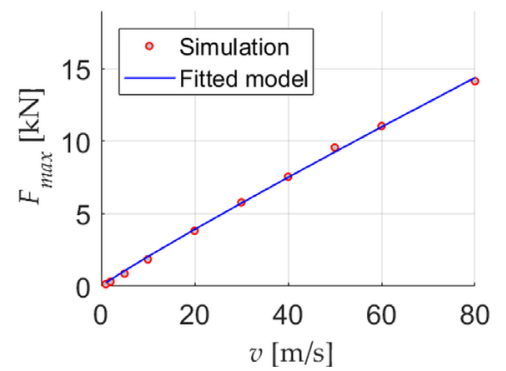

Fig. 9 The maximal contact force as the function of the velocity of the sphere before the impact

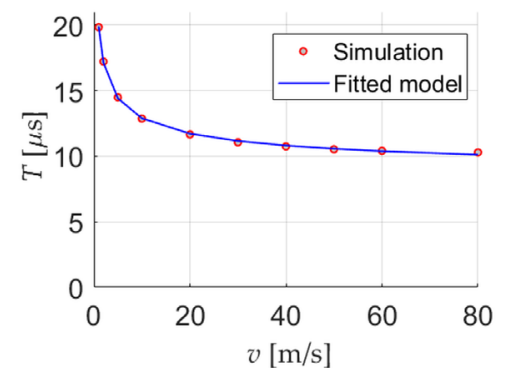

Fig. 10 The contact time as the function of the velocity of the sphere before the impact

\section{Comparison of the results for different material model pairs}

The simulations were carried out with different material model pairs providing opportunity to investigate the applicability of different material model pairs. The compared characteristics can be seen in Fig. 11.

The contact force is the maximum for the E-R model, whereas the EP-EP model results the minimum contact force for the investigated models. The result for the E-E model lies in between the E-R and EP-EP models. Similar tendency can be observed for the contact time as well. However, at smaller velocities the contact time can be larger for the EP-EP model than for the E-E model.
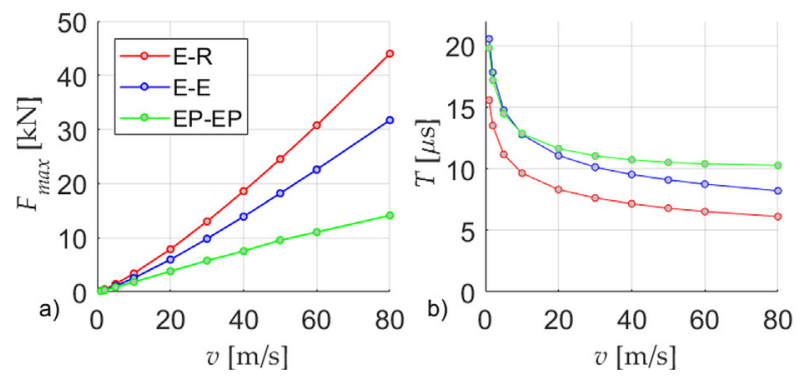

Fig. 11 The a) maximal contact force and b) contact time as the function of the velocity of the sphere before the impact compared in case of the three investigated material models

\section{The effect of the friction coefficient}

During the simulations four different friction coefficients were investigated, namely the values $0,0.1,0.4$ and 0.8 . The obtained results can be seen in Fig. 12 for the most complex EP-EP model.

As the figure clearly shows, the effect of the friction coefficient is negligible in the investigated parameter range. The effect of the friction coefficient was also investigated in case of two different impact angles, namely $30^{\circ}$ and $60^{\circ}$. As in case of the normal impact, the effect of the friction coefficient was also negligible. Here, only the frictionless case was compared with the case using value 0.8 for the friction coefficient. The comparisons are shown in Figs. 13 and 14.
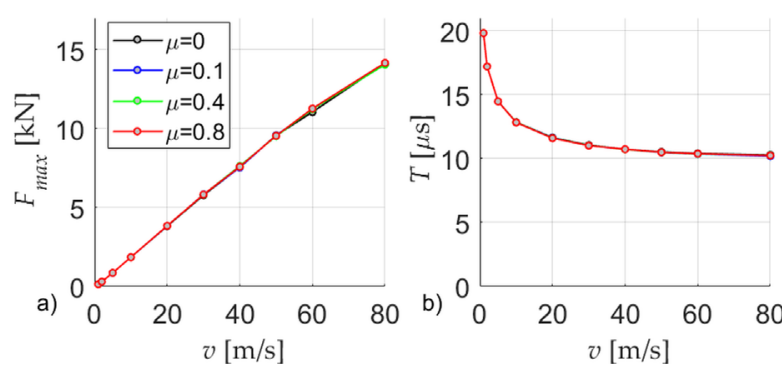

Fig. 12 The investigated contact characteristics as the function of the velocity of the sphere before the impact in case of different friction coefficients
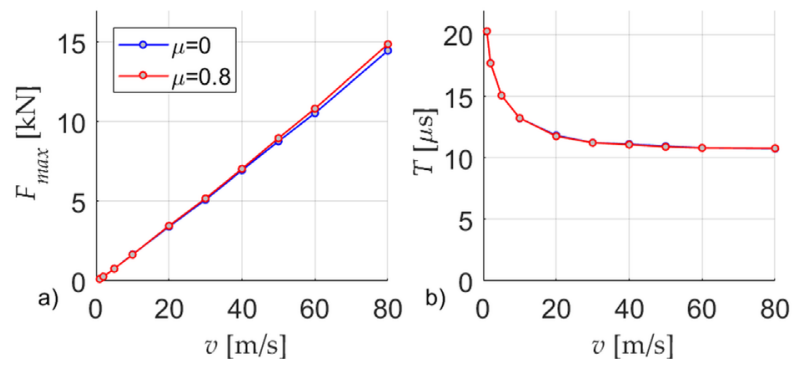

Fig. 13 The effect of the friction on the investigated contact characteristics in case of the $30^{\circ}$ angle of impact
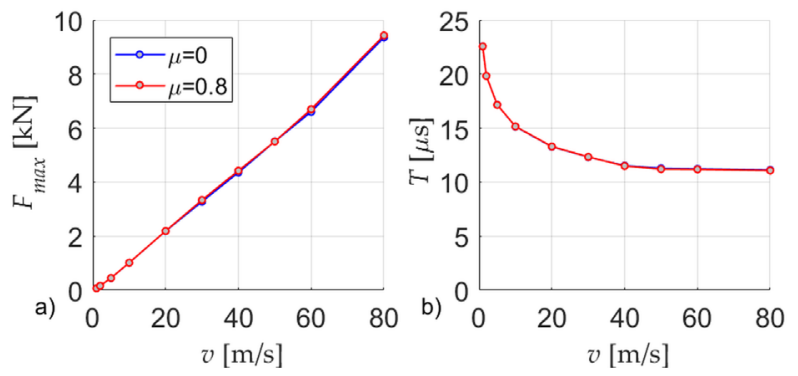

Fig. 14 The effect of the friction on the investigated contact characteristics in case of the $60^{\circ}$ angle of impact 
Furthermore, the effect of the friction coefficient was also investigated in case of the normal impact of an elastic sphere and rigid wall using two different friction coefficient values. The obtained results are presented in Fig. 15. According to the simulations, the friction does not affect considerably the contact time. However, it causes a minor change in the maximal contact force.

\section{The effect of the angle of impact}

The contact characteristics were investigated at five different angles of the impact, which was defined according to Fig. 16. The simulations were carried out for the EP-EP model. Based on the conclusions made in the previous sub-section, only frictionless impact was investigated.

The obtained characteristics can be seen in Fig. 17. According to the results, the contact time slightly increases as the angle of impact increases. However, the maximal contact force depends considerably on the angle of impact. At larger angles the maximum contact force decrease is more visible compared to the normal impact even at smaller velocity magnitudes.

As the figure shows the maximal contact force decreases with the increase of the impact angle.
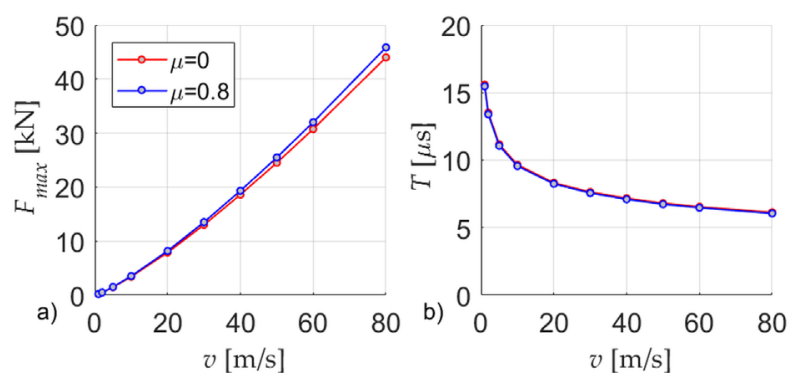

Fig. 15 The investigated contact characteristics as the function of the velocity of the sphere before the impact in case of the E-R material model for two different values of the friction coefficient

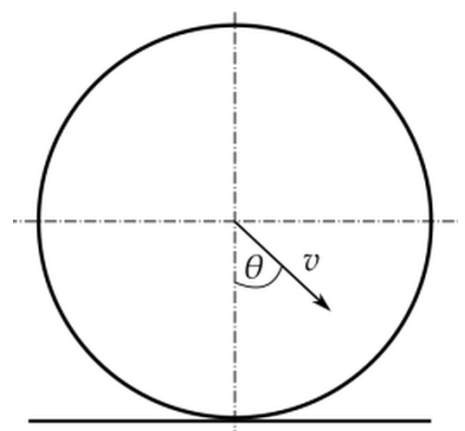

Fig. 16 The definition of the angle of the impact
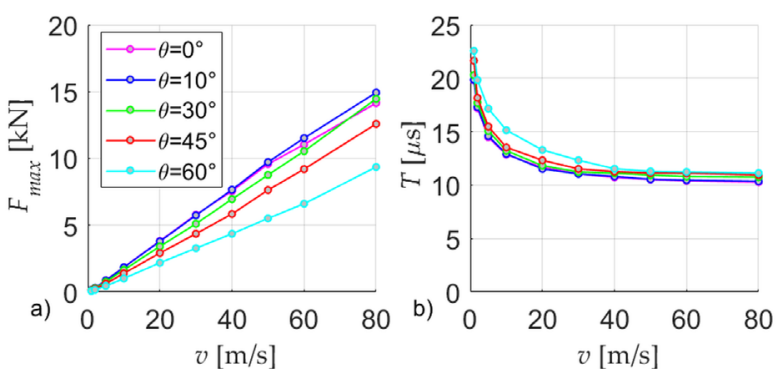

Fig. 17 The investigated contact characteristics as the function of the velocity of the sphere before the impact in case of different angles of impact

\section{Conclusions}

The impact characteristics of a solid sphere and a flat wall were investigated in this paper. The contact force value and the contact time were the main targets to analyze in terms of the initial velocity of the solid sphere, whereas the wall was fixed. In order to reveal the effect of the applied material model on the results, different material model pairs were used in the analyses.

A comparison of the finite element models and the existing models was presented and a mesh independency investigation was also carried out. A wide range of parameters were considered during the study. The relationship of the initial velocity magnitude of the sphere before impact and the investigated contact characteristics, namely the maximal contact force and the contact time, was presented for each of the explored material pairs and fixed geometrical models. The results for different material models were compared.

Moreover the effect of the friction coefficient and the angle of impact were also investigated. As the results showed, the friction coefficient has a minor effect on the impact for the investigated cases. The simulation of the oblique impact showed that for higher impact velocities and greater impact angles the angle has a significant effect on the examined parameters, mainly on the maximal contact force.

\section{Acknowledgement}

The support from the National Research, Development and Innovation Office Hungary (Project Identifier: FK 128662) is gratefully acknowledged. The research reported in this paper has been supported by the National Research, Development and Innovation Fund (TUDFO/51757/2019ITM, Thematic Excellence Program). This project has 
received funding from the European Research Council (ERC) under the European Union's Horizon 2020 research and innovation programme (Grant agreement No. 862308).

\section{References}

[1] Shigley, J. E., Mischke, C. R. "Mechanical Engineering Design", McGraw-Hill, New York, NY, USA, 1989.

[2] Johnson, K. L. "One Hundred Years of Hertz Contact", Proceedings of the Institution of Mechanical Engineers, 196(1), pp. 363-378, 1982. https://doi.org/10.1243/PIME_PROC_1982_196_039_02

[3] Hertz, H. "Ueber die Berührung fester elastischer Körper" (About the Contact of Solid Elastic Bodies), Journal für die reine und angewandte Mathematik, 1882(92), pp. 156-171, 1882. (in German) https://doi.org/10.1515/crll.1882.92.156

[4] Hunter, S. C. "Energy Absorbed by Elastic Waves during Impact", Journal of the Mechanics and Physics of Solids, 5(3), pp. 162-171, 1957.

https://doi.org/10.1016/0022-5096(57)90002-9

[5] Reed, J. "Energy losses due to elastic wave propagation during an elastic impact", Journal of Physics D: Applied Physics, 18(12), pp. 2329-2337, 1985. https://oi.org/10.1088/0022-3727/18/12/004

[6] Goldsmith, W. "Impact: The Theory and Physical Behaviour of Colliding Solids", Edward Arnold Ltd, London, England, 1960.

[7] Hunt, K. H., Crossley, F. R. E. "Coefficient of Restitution Interpreted as Damping in Vibroimpact", Journal of Applied Mechanics, 42(2), pp. 440-445, 1975. https://doi.org/10.1115/1.3423596

[8] Gonthier, Y., McPhee, J., Lange, C., Piedbœuf, J.-C. "A Regularized Contact Model with Asymmetric Damping and Dwell-Time Dependent Friction", Multibody System Dynamics, 11(3), pp. 209-233, 2004. https://doi.org/10.1023/B:MUBO.0000029392.21648.bc

[9] Flores, P., Machado, M., Silva, M. T., Martins, J. M. "On the continuous contact force models for soft materials in multibody dynamics", Multibody System Dynamics, 25(3), pp. 357-375, 2011. https://doi.org/10.1007/s11044-010-9237-4
[10] Hardy, C., Baronet, C. N., Tordion, G. V. "The elastoplastic indentation of a half-space by a rigid sphere", International Journal for Numerical Methods in Engineering, 3(4), pp. 451-462, 1971. https://doi.org/10.1002/nme.1620030402

[11] Follansbee, P. S., Sinclair, G. B. "Quasi-static Normal Indentation of an Elasto-plastic Half-space by a Rigid Sphere-I: Analysis", International Journal of Solids and Structures, 20(1), pp. 81-91, 1984. https://doi.org/10.1016/0020-7683(84)90078-7

[12] Thornton, C. "Coefficient of Restitution for Collinear Collisions of Elastic-Perfectly Plastic Spheres", Journal of Applied Mechanics, 64(2), pp. 383-386, 1997. https://doi.org/10.1115/1.2787319

[13] Thornton, C., Ning, Z. "A theoretical model for the stick/ bounce behaviour of adhesive, elastic-plastic spheres", Powder Technology, 99(2), pp. 154-162, 1998. https://doi.org/10.1016/S0032-5910(98)00099-0

[14] Ghaednia, H., Pope, S. A., Jackson, R. L., Marghitu, D. B. "A comprehensive study of the elasto-plastic contact of a sphere and a flat", Tribology International, 93, pp, 78-90, 2016. https://doi.org/10.1016/j.triboint.2015.09.005

[15] Berezvai, Sz., Kossa, A., Bachraty, D., Stepan, G. "Numerical and experimental investigation of the applicability of pellet impacts for impulse excitation", International Journal of Impact Engineering, 115, pp. 19-31, 2018. https://doi.org/10.1016/j.ijimpeng.2018.01.006

[16] NSK "Bearing materials", CAT. No. E728g 2013 E-10, [pdf] NSK, Brasil, Available at: http://www.nsk.com.br/upload/file/nsk_cat_ e728g_10.pdf [Accessed: 15 December 2019]

[17] Dassault Systemes "Abaqus, (Version 2016)", [computer program] Available at: https://www.3ds.com/products-services/simulia/ products/abaqus [Accessed: 15 December 2019] 